\title{
The Predictive Value of Insulin-Like Growth Factor 1 in Irradiation-Dependent Growth Hormone Deficiency in Childhood Cancer Survivors
}

\author{
Alessandro Cattoni $^{a}$ Enrico Clarke $^{b}$ Assunta Albanese ${ }^{a}$ \\ a Department of Paediatric Endocrinology, Royal Marsden NHS Foundation Trust, London, UK; \\ ${ }^{b}$ Department of Radiotherapy, Royal Marsden NHS Foundation Trust, London, UK
}

\section{Keywords}

Insulin-like growth factor 1 - Growth · Hypopituitarism .

Survivor · Neoplasm

\begin{abstract}
Background: The literature contains conflicting reports on the value of low insulin-like growth factor 1 (IGF-1) levels in predicting radiation-induced growth hormone $(\mathrm{GH})$ deficiency (GHD) in childhood cancer survivors (CCS). These reports often involve small samples of patients who have received irradiation or mixed cohorts including non-irradiated subjects. Objective: We undertook an analysis of the predictive value of low IGF-1 in CCS at risk for GHD after cranial radiotherapy involving the hypothalamic-pituitary (HP) area in a large single-centre cohort. Methods: We performed a retrospective analysis on 158 CCS diagnosed with GHD between January 1, 2003 and October 31, 2017 and identified 117 patients who received radiation for tumours not directly involving the HP area. Results: In this cohort, IGF-1 levels $<-2$ standard deviation scores (SDS) had a sensitivity of $31.9 \%$ for GHD; however, they were statistically more frequent ( $p=0.0023$ ) and had a higher sensitivity (45.6\%) among patients with severe GHD. At final height reassessment, IGF-1 <-2 SDS had a sensitivity of $35.0 \%$ for GHD, but a positive predictive value of $100 \%$. Finally, pretreatment
\end{abstract}

\section{KARGER}

(c) 2019 S. Karger AG, Basel

E-Mail karger@karger.com

www.karger.com/hrp
IGF-1 values showed no correlation with the number of impaired pituitary hormonal axes in patients with multiple pituitary deficiencies. Conclusions: IGF-1 levels <-2 SDS showed a low sensitivity at predicting radiation-induced GHD both in childhood and in adulthood, but a high positive predictive value for $\mathrm{GH}$ status at final height reassessment.

(c) 2019 S. Karger AG, Basel

\section{Introduction}

Many of the endocrine late effects experienced by childhood cancer survivors (CCS) are the result of some degree of neuroendocrine impairment involving the hypothalamic-pituitary (HP) area as a late consequence of either antineoplastic treatment of the childhood cancer or direct involvement by adjacent brain tumours. The detrimental effect of radiotherapy on HP function has been widely described and occurs when the hypothalamus and/or pituitary fall within the radiation field [1-4].

The incidence of pituitary hormone deficiencies depends on the radiation biological effective dose involving the HP area, and a well-defined progressive involvement is described for increasingly higher radiation doses, with somatotrophic cells being most sensitive to radiation-induced damage. Irradiation-dependent growth hormone 
(GH) deficiency (GHD) is therefore the most frequent pituitary dysfunction described in CCS, as it occurs also for low exposure of the HP area to radiation (10-18 Gy) [5-7].

Low insulin-like growth factor 1 (IGF-1) levels provide supporting evidence for performing dynamic $\mathrm{GH}$ testing in patients with suggestive auxological data and/or previous medical history. However, conflicting evidence has been reported about the value of low IGF-1 levels in predicting radiation-induced GHD in CCS, with some studies supporting a poor diagnostic impact in this specific population [8-10] and others demonstrating a satisfactory role as a screening tool also in irradiated CCS [11, 12]. Most of these outcomes are drawn from small samples of patients and do not provide specific figures about the distribution of IGF-1 values in relation to the severity of GHD and the radiation exposure of the HP area.

We performed a retrospective analysis in our oncology centre in order to assess the screening role of IGF- 1 levels both at the diagnosis of childhood GHD and at final height retesting for GH status in a selected cohort of irradiated leukaemia and brain tumour survivors. In addition, we correlated the distribution of IGF-1 values with severity of GHD, pituitary radiation exposure, and number of pituitary hormonal deficiencies.

\section{Patients and Methods}

\section{Patients}

A total of 158 CCS (64 females, 94 males) were diagnosed with GHD and started on recombinant human GH (rhGH) between January 1, 2003 and October 31, 2017 at the Royal Marsden Hospital in Sutton, London. Their mean age at the moment of data collection was $17.86 \pm 5.08$ years.

The recorded oncological diagnosis was brain tumour for $123 / 158$ subjects $(77.8 \%) ; 29 / 158$ patients $(18.4 \%)$ were diagnosed with leukaemia and received cranial irradiation as either treatment for central nervous system involvement or as part of total body irradiation (TBI) conditioning protocols before bone marrow transplantation; $6 / 158$ patients $(3.80 \%)$ were diagnosed with a rhabdomyosarcoma involving cranial extracentral nervous system structures (orbit, nasopharynx, middle ear, and infratemporal fossa) and treated with local radiotherapy. A total of 141 of the 158 subjects received either cranial irradiation, craniospinal irradiation, or $\mathrm{TBI}$, and in all these patients the HP area fell within the irradiation field. Table 1 shows the general features of the population enrolled in our study.

In order to describe the specific impact of irradiation on GHD, we selected a subcohort of 117 irradiated patients diagnosed with tumours not directly involving the HP area (RT-NHP group). Patients with craniopharyngiomas, germ cell tumours involving the pituitary gland, optic gliomas with direct involvement of the hypothalamus or radiological evidence of compression on the pituitary gland, and one patient with pituitary stalk thickening were excluded from the RT-NHP group.

IGF-1 in Radiation-Induced Growth Hormone Deficiency

\section{Methods}

The present study was a single-centre, observational, retrospective analysis. All CCS diagnosed with GHD between January 1, 2003 and October 31, 2017 before the age of 16 years were eligible. Patients with an annual linear growth velocity $<2 \mathrm{~cm}$ per year, clinical or laboratory data suggestive of hepatic or renal impairment at the moment of IGF-1 assessment, syndromic conditions related to GHD, and/or poorly controlled pituitary insufficiencies of axes other than GH were excluded from the study.

Definitions. Our laboratory employed four different assays to assess IGF-1 levels from January 1, 2003 to October 31, 2017: Nichols Advantage ${ }^{\circledR}$ (Nichols Institute Diagnostics), Immulite $2000^{\circledR}$ (Siemens), iSYS ${ }^{\circledR}$ (Immunodiagnostic Systems), and Liai$\operatorname{son}^{\circledR}$ (DiaSorin). Given this heterogeneity, we recorded IGF-1 values as standard deviation scores (IGF-1 SDS) according to age- and sex-adjusted reference ranges for each kit. In our cohort of CCS, the diagnosis of GHD in childhood was confirmed by the finding of a $\mathrm{GH}$ peak value $<7 \mu \mathrm{g} / \mathrm{L}$ after a single standardized stimulation test. Although two distinct dynamic tests are generally required to diagnose GHD, a single pathological test was considered sufficient in case of either a defined central nervous system disease, a previous history of cranial radiation, or the presence of multiple pituitary hormone deficiencies, according to the Consensus Guidelines of the Growth Hormone Research Society [13] and the UK National Institute for Health and Clinical Excellence diagnostic criteria [14]. GHD was recorded as severe if the GH peak value achieved after a single stimulation test was $<3 \mu \mathrm{g} / \mathrm{L}$ and as partial if the $\mathrm{GH}$ peak ranged from 3 to $6.99 \mu \mathrm{g} / \mathrm{L}$. When a GH status reassessment was performed at final height in teenagers and young adults, GHD was confirmed by a peak value $\leq 5 \mu \mathrm{g} / \mathrm{L}$. Again, GHD at final height was considered as severe for peaks $<3 \mu \mathrm{g} / \mathrm{L}$ and as partial for peaks between 3 and $4.99 \mu \mathrm{g} / \mathrm{L}$. Insulin tolerance test (ITT) was the test of choice in diagnosing radiation-induced GHD for all our patients. Whenever a concurrent clinical condition contraindicated ITT, a glucagon test was performed.

Estimation of the Pituitary Exposure to Radiation. For all the patients included in the dosimetric analysis, the approved radiation plan was obtained and the pituitary gland was delineated on each axial slice. Mean, maximum, and minimum doses in Gy were recorded. Contours were created within the Philips Pinnacle ${ }^{\circledR}$ v9.10 treatment planning system (Philips Medical Systems, Fitchburg, WI, USA).

Statistics. Results are expressed as mean \pm 1 standard deviation. Descriptive statistics were used to describe the diagnostic accuracy of IGF-1. Statistical comparisons between subgroups of patients were performed with the Student $t$ test after confirming a normal distribution of the selected variables with the MannWhitney $U$ test. $\chi^{2}$ test/Fisher exact test and Pearson coefficient were used to assess the statistical association and the statistical correlation between unrelated variables, respectively. We used linear regression analysis to analyse the impact of increasing pituitary radiotherapy doses on GH peaks achieved. Multinomial logistic regression analysis provided an estimation of the contribution of several independent variables on the distribution of IGF-1 SDS. The SAS JMP Software ${ }^{\circledR}$ was used to perform the statistical analyses. 
Table 1. Anatomical site involved by the tumour, frequency, mean age, and age range at diagnosis for each oncological histotype

\begin{tabular}{|c|c|c|c|c|c|}
\hline Histotype & Anatomical site involved & & $\begin{array}{l}\text { Patients, } \\
n(\%)\end{array}$ & $\begin{array}{l}\text { Mean age at } \\
\text { diagnosis } \pm S D\end{array}$ & $\begin{array}{l}\text { Age range } \\
\text { at diagnosis }\end{array}$ \\
\hline Medulloblastomas & posterior fossa & & $44(27.8 \%)$ & $7.24 \pm 3.01$ & $1.69-12.78$ \\
\hline Gliomas & $\begin{array}{l}\text { optic pathway } \\
\text { hypothalamus } \\
\text { thalamus } \\
\text { tectal plate } \\
\text { brainstem } \\
\text { pineal region } \\
\text { cerebellum }\end{array}$ & $\begin{array}{r}17 \\
2 \\
7 \\
2 \\
7 \\
1 \\
1\end{array}$ & $37(23.4 \%)$ & $5.33 \pm 2.84$ & $0.61-14.17$ \\
\hline Craniopharyngiomas & HP area & & $20(12.7 \%)$ & $9.26 \pm 3.20$ & $2.45-14.25$ \\
\hline Germ cell tumours & $\begin{array}{l}\text { pineal region } \\
\text { HP area }\end{array}$ & $\begin{array}{l}3 \\
5\end{array}$ & $8(5.1 \%)$ & $10.00 \pm 2.57$ & $5.39-12.33$ \\
\hline Ependymomas & posterior fossa & & $4(2.5 \%)$ & $4.29 \pm 3.09$ & $1.00-8.19$ \\
\hline Pineoblastomas & pineal gland & & $3(1.9 \%)$ & NA & $1.78-10.70$ \\
\hline Rhabdomyosarcomas & $\begin{array}{l}\text { middle ear } \\
\text { nasopharynx } \\
\text { infratemporal fossa } \\
\text { orbit }\end{array}$ & $\begin{array}{l}2 \\
2 \\
1 \\
1\end{array}$ & $6(3.8 \%)$ & $5.76 \pm 2.21$ & $4.27-9.86$ \\
\hline Teratoid rhabdoid tumour & lateral hemispheres & & $1(0.6 \%)$ & NA & NA \\
\hline Primitive neuroectodermal tumor & thalamus & & $1(0.6 \%)$ & NA & NA \\
\hline Carcinoma & choroid plexus & & $1(0.6 \%)$ & NA & NA \\
\hline Desmoplastic round cell tumour & infratemporal fossa & & $1(0.6 \%)$ & NA & NA \\
\hline Atypical teratoma & thalamus & & $1(0.6 \%)$ & NA & NA \\
\hline Pituitary stalk thickening & HP area & & $1(0.6 \%)$ & NA & NA \\
\hline Non-Hodgkin lymphoma & lateral hemispheres & & $1(0.6 \%)$ & NA & NA \\
\hline Leukaemias & NA & & $29(18.4 \%)$ & $5.07 \pm 2.52$ & $1.77-10.33$ \\
\hline
\end{tabular}

HP, hypothalamic-pituitary; NA, not applicable; SD, standard deviation.

\section{Results}

\section{GH Stimulation Test at GHD Diagnosis}

All the enrolled 158 patients underwent a GH stimulation test and results were available in $153 / 158$ patients. In Table 2, means, standard deviation, and range of the GH peak values are represented in each oncological subgroup. Mean GH peak values were statistically lower in patients diagnosed with craniopharyngioma than in subjects with leukaemia ( $p<0.001)$, medulloblastoma $(p<0.01)$, optic pathway glioma $(p=0.04)$, and extraoptic glioma $(p<$ 0.01 ). In the whole study population, the $\mathrm{GH}$ stimulation test was performed $1.83 \pm 1.15$ years (range $0.02-5.24$ ) and rhGH replacement therapy was started $2.12 \pm 1.15$ years (range 0.17-5.43) after the end of antineoplastic treatment. In patients with craniopharyngioma, both $\mathrm{GH}$ testing and rhGH treatment were undertaken earlier than in patients with other oncological conditions $(0.38 \pm 0.44$ and $0.62 \pm 0.48$ years, respectively).

In Table 3, we report the mean chronological age at the moment of GH testing and when rhGH was started both for the whole study population and for specific oncological subcohorts. In addition, it shows the average time between the end of anticancer treatment and the start of hormonal replacement treatment. 
Table 2. GH peak levels after GH stimulation tests in the whole population and the diagnostic subgroups

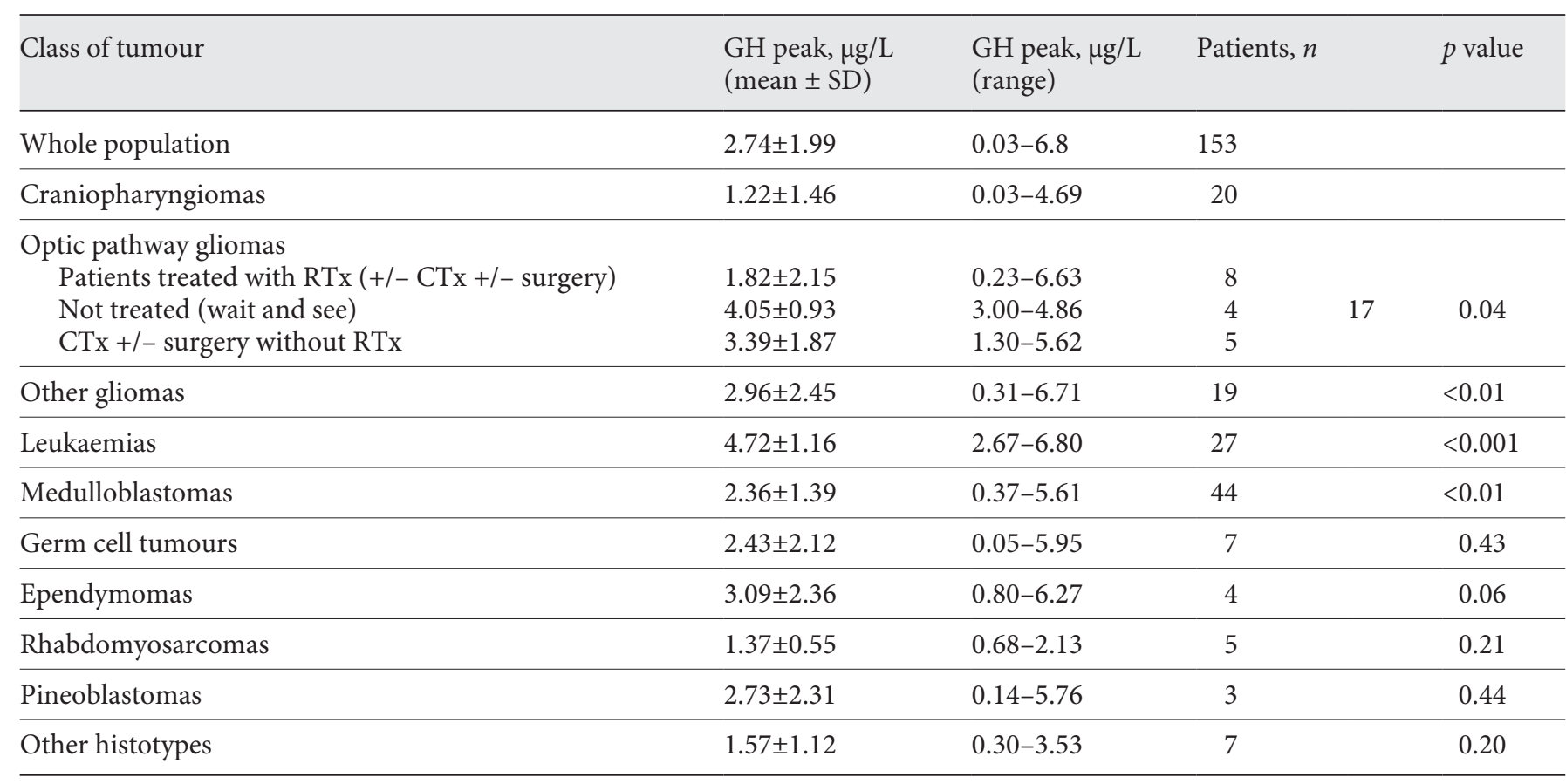

In the last column on the right, the $p$ values of the statistical comparisons between $\mathrm{GH}$ peaks for each oncological subclass and GH peaks in craniopharyngiomas are provided. CTx, chemotherapy; GH, growth hormone; RTx, radiotherapy; SD, standard deviation.

Table 3. Mean chronological age at GH testing and at start of rhGH therapy in the whole population and in selected oncological subcohorts as well as time between end of antineoplastic treatment and start of rhGH therapy

\begin{tabular}{|c|c|c|c|c|c|}
\hline \multirow[t]{2}{*}{ Class of tumour } & \multicolumn{2}{|c|}{ Age at GH testing, years } & \multicolumn{2}{|c|}{ Age at rhGH start, years } & \multirow{2}{*}{$\begin{array}{l}\text { Time between end } \\
\text { of antineoplastic } \\
\text { treatment and start } \\
\text { of rhGH, years }\end{array}$} \\
\hline & mean $\pm \mathrm{SD}$ & range & mean $\pm S D$ & range & \\
\hline Whole population & $10.05 \pm 2.71$ & $2.33-16.20$ & $10.44 \pm 2.67$ & $3.75-16.48$ & $2.12 \pm 1.15$ \\
\hline Craniopharyngiomas & $10.44 \pm 2.70$ & $5.51-14.83$ & $10.68 \pm 2.71$ & $5.87-15.13$ & $0.62 \pm 0.47$ \\
\hline Gliomas & $9.41 \pm 2.59$ & $2.33-15.70$ & $10.27 \pm 2.58$ & $4.09-15.92$ & $2.48 \pm 1.13$ \\
\hline Leukaemias & $10.45 \pm 1.87$ & $6.07-14.56$ & $10.72 \pm 1.99$ & $6.50-14.92$ & $3.08 \pm 1.12$ \\
\hline Medulloblastomas & $9.91 \pm 2.62$ & $5.14-14.92$ & $10.27 \pm 2.54$ & $4.09-15.92$ & $2.34 \pm 0.58$ \\
\hline Pineal tumours (GCTs/pineoblastomas) & $10.89 \pm 3.35$ & $5.41-16.15$ & $11.14 \pm 3.38$ & $5.56-16.48$ & $1.95 \pm 0.57$ \\
\hline Other histotypes & $9.59 \pm 3.44$ & $3.63-16.20$ & $9.78 \pm 3.36$ & $3.75-16.31$ & $2.59 \pm 1.17$ \\
\hline
\end{tabular}

GCTs, germ cell tumors; GH, growth hormone; rhGH, recombinant human growth hormone; SD, standard deviation.

\section{Sensitivity of Low IGF-1 and Insulin-Like Growth}

Factor-Binding Protein 3 Levels

Both IGF-1 levels and GH peaks were available for $151 / 158$ patients from the whole population and for $113 / 117$ from the RT-NHP group. Table 4 shows the distribution of IGF-1 SDS values in the different subgroups of GHD patients. As expected, the sensitivity for IGF-1 values $<-2$ SDS was higher in irradiated patients with tumours involving the HP area than in the RT-NHP cohort and higher in irradiated than non-irradiated patients, although the difference was not statistically significant in both cases $\left(\chi^{2}=2.15, p=0.14\right.$, and $\chi^{2}=1.96, p=0.16$, respectively). In the RT-NHP cohort, IGF-1 sensitivity for values $<-2$ SDS was $31.86 \%$. 
Table 4. IGF-1 distribution in relation to different patients subgroups

\begin{tabular}{|c|c|c|c|c|c|}
\hline Irradiated patients with tumours not involving the HP area & $36(31.86 \%)$ & $65(57.52 \%)$ & $10(8.85 \%)$ & $2(1.77 \%)$ & 113 \\
\hline Irradiated patients with tumours involving the HP area & $11(47.83 \%)$ & $12(52.17 \%)$ & $0(0.00 \%)$ & $0(0.00 \%)$ & 23 \\
\hline
\end{tabular}

HP, hypothalamic-pituitary; IGF-1, insulin-like growth factor 1; SDS, standard deviation scores.

Table 5. Distribution of IGF-1 values in relation to different oncological diagnoses and pituitary radiation exposure for each tumoral class

\begin{tabular}{|c|c|c|c|c|c|c|c|}
\hline Tumour class & $\begin{array}{l}\text { IGF-1 } \\
<-2 \text { SDS }\end{array}$ & $\begin{array}{l}\text { IGF-1 } \\
\geq-2 \text { and } \\
<-1 \text { SDS }\end{array}$ & $\begin{array}{l}\text { IGF- } 1 \\
\geq-1 \text { and } \\
<1 \text { SDS }\end{array}$ & $\begin{array}{l}\text { IGF-1 } \\
\geq 1 \text { SDS }\end{array}$ & $\begin{array}{l}\text { Patients } \\
\text { with avail- } \\
\text { able IGF-1 }\end{array}$ & $\begin{array}{l}\text { Radiation exposure } \\
\text { to the pituitary, Gy } \\
\text { (mean } \pm \mathrm{SD} \text {, range) }\end{array}$ & $p$ value \\
\hline Irradiated medulloblastomas & $18(43.90 \%)$ & $16(39.02 \%)$ & $7(17.07 \%)$ & $0(0.00 \%)$ & 41 & $41.3 \pm 7.4(27.2-55.12)$ & 0.014 \\
\hline Irradiated craniopharyngiomas & $9(52.94 \%)$ & $7(41.18 \%)$ & $1(5.88 \%)$ & $0(0.00 \%)$ & 17 & $51.8 \pm 2.4(50.0-56.9)$ & 0.009 \\
\hline All leukaemias & $5(17.86 \%)$ & $16(57.14 \%)$ & $7(25.00 \%)$ & $0(0.00 \%)$ & 28 & $19.5 \pm 6.7(14.4-38.4)$ & 0.64 \\
\hline $\begin{array}{l}\text { Leukaemias treated with } \geq 18 \text { Gy } \\
\quad \text { (TBI + cranial boost/CNS } \\
\text { involvement) }\end{array}$ & $4(28.57 \%)$ & $7(50.00 \%)$ & $3(21.43 \%)$ & $0(0.00 \%)$ & 14 & $24.6 \pm 6.1(18-38.4)$ & 0.14 \\
\hline $\begin{array}{l}\text { Leukaemias treated with } 14.4 \mathrm{~Gy} \\
\quad \text { (only TBI) }\end{array}$ & $1(7.14 \%)$ & $9(64.29 \%)$ & $4(28.57 \%)$ & $0(0.00 \%)$ & 14 & 14.4 & 1 \\
\hline
\end{tabular}

In the column on the right, we report the $p$ values between the sensitivity of low IGF-1 in each oncological subclass versus the sensitivity in leukaemic patients exposed to only 14.4 Gy of TBI. CNS, central nervous system; IGF-1, insulin-like growth factor 1; SD, standard deviation; SDS, standard deviation scores; TBI, total body irradiation.

Table 5 provides figures about those patients from the four most represented diagnostic subgroups (medulloblastomas, craniopharyngiomas, leukaemias, and gliomas) who were exposed to irradiation involving the HP area as part of their antineoplastic treatment. In particular, it represents the distribution of IGF-1 values according to their oncological diagnosis and the pituitary radiation exposure for each tumoral subclass. IGF-1 sensitivity for values $<-2$ SDS was statistically lower in leukaemic subjects who received an HP radiation dose of 14.4 Gy than irradiated patients diagnosed with medulloblastoma (HP radiation dose always $>24 \mathrm{~Gy}, p=0.014$ as per twotailed Fisher exact test) and subjects with craniopharyngiomas $(p=0.009)$.

The frequency of IGF-1 values $<-2$ SDS was statistically higher in patients with severe GHD (GH peak $<3 \mu \mathrm{g} / \mathrm{L}$ ) than in subjects with partial GHD (GH peak $\geq 3 \mu \mathrm{g} / \mathrm{L})$ both in our whole population $\left(\chi^{2}=14.31, p=\right.$ $0.0002)$ and in the RT-NHP group $\left(\chi^{2}=8.65, p=0.003\right)$, as shown in Table 6 . In the RT-NHP cohort, we found a positive but weak statistical correlation between baseline IGF-1 SDS values and GH peaks after dynamic tests (Pearson coefficient $0.31, p<0.001$ ). Insulin-like growth factor-binding protein 3 (IGFBP-3) levels were available in 95/117 patients from the RT-NHP cohort. In this selected group, IGFBP-3 levels $<-2$ SDS had a sensitivity of $7.37 \%$ (7/95 patients).

\section{Correlation between Pituitary Radiation Dose and GH Peak and IGF-1 SDS}

An estimation of the pituitary exposure to radiation was available in 77/117 subjects from the RT-NHP cohort. In this group, we found a significant negative correlation between the pituitary dose and the GH peaks 
Table 6. Contingency table for the distribution of IGF-1 levels in relation to the severity of GHD in the whole study population and in the RT-NHP group

\begin{tabular}{|c|c|c|c|c|c|c|}
\hline \multirow[t]{2}{*}{ Severity of GHD } & \multicolumn{2}{|c|}{ IGF-1 $<-2$ SDS } & \multicolumn{2}{|c|}{ IGF-1 $\geq-2$ SDS } & \multicolumn{2}{|c|}{ Total } \\
\hline & WP & RT-NHP & WP & RT-NHP & WP & RT-NHP \\
\hline Severe GHD (peak <3 $\mu \mathrm{g} / \mathrm{L})$ & 39 & 27 & 46 & 35 & 85 & 62 \\
\hline Partial GHD (peak $\geq 3 \mu \mathrm{g} / \mathrm{L})$ & 11 & 9 & 55 & 42 & 66 & 51 \\
\hline Total & 50 & 36 & 101 & 77 & 151 & 113 \\
\hline
\end{tabular}

GHD, growth hormone deficiency; IGF-1, insulin-like growth factor 1; RT-NHP group, subcohort of 117 irradiated patients diagnosed with tumours not directly involving the hypothalamic-pituitary area; SDS, standard deviation scores; WP, whole population.

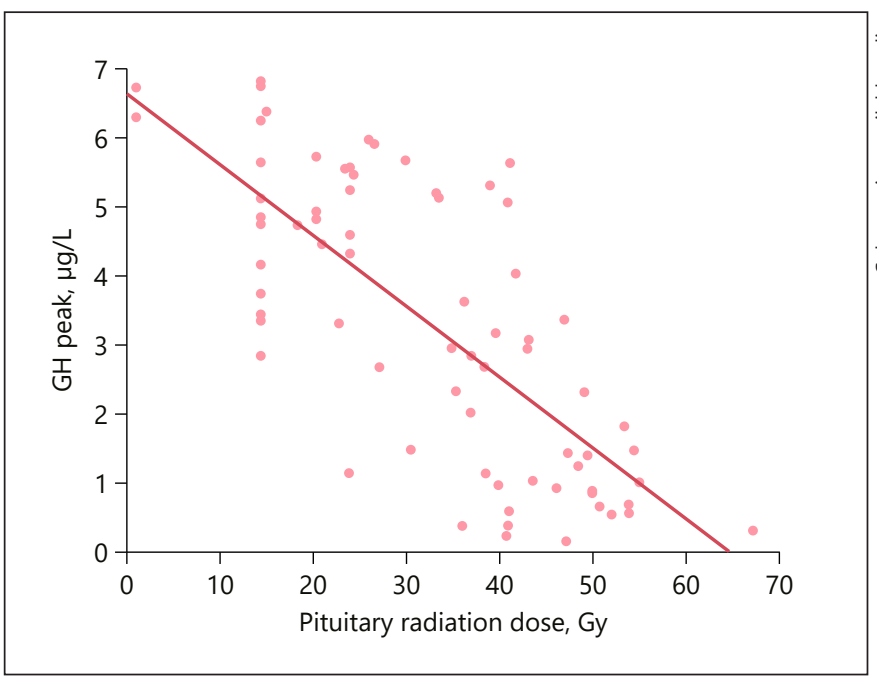

Fig. 1. Linear regression of pituitary dose ( $x$ axis) and GH peak ( $y$ axis): $y=6.63-0.1 x ; r=-0.75, p<0.001$. GH, growth hormone.

achieved after GH stimulation test in childhood (Pearson coefficient $-0.75, p=0.00001$ ). Figure 1 shows the linear regression between the radiation dose to the pituitary gland and the peak achieved after GH stimulation tests in childhood. According to this model, the GH peak drops by an average value of $0.1 \mu \mathrm{g} / \mathrm{L}$ for each additional Gy of pituitary exposure to radiation $\left(p<0.0001, R^{2}=0.55\right)$.

Our results also showed a weak but statistically significant negative correlation between pituitary radiation dose and IGF-1 SDS values in the RT-NHP cohort (Pearson coefficient $-0.32, p=0.006$ ). Figure 2 represents the linear regression model between independent (radiation dose) and dependent (IGF-1 SDS) variables.

IGF-1 in Radiation-Induced Growth Hormone Deficiency

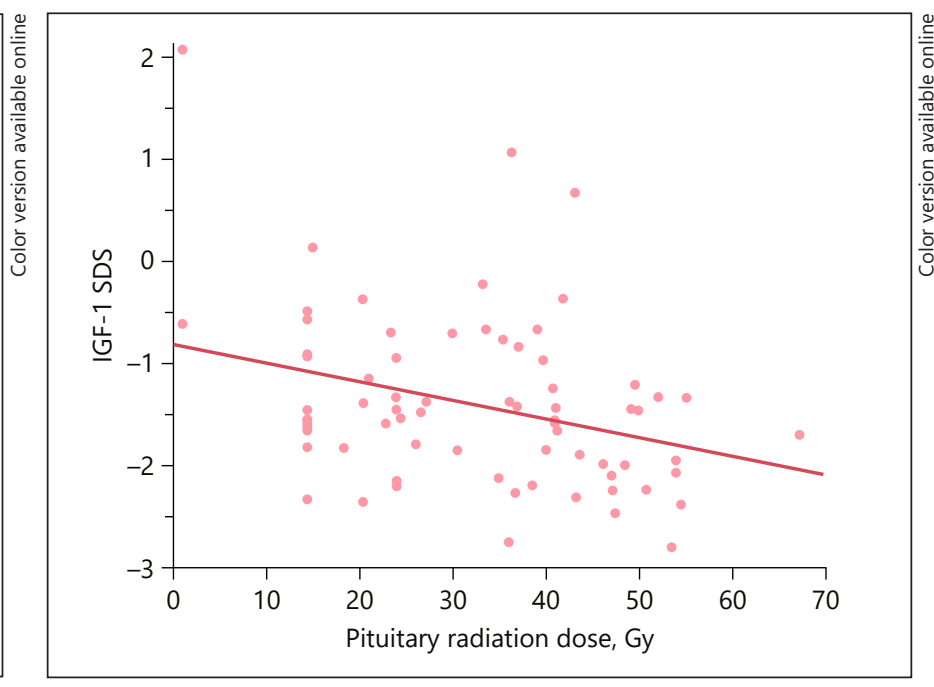

Fig. 2. Linear regression of pituitary dose ( $x$ axis) and IGF-1 SDS ( $y$ axis): $y=-0.83-0.02 x ; r=-0.32, p=0.006$. IGF-1, insulin-like growth factor 1; SDS, standard deviation score.

\section{The Impact of Different Variables on IGF-1 \\ Distribution}

We performed a multinomial logistic regression analysis to assess the impact of RT-NHP patients' demographic (sex, age), auxological (height SDS, BMI SDS), and treatment features (pituitary radiation dosimetry, time between the end of radiotherapy and GH testing) on the distribution of baseline IGF-1 SDS levels at diagnosis of GHD.

The only independent variable with a statistically remarkable impact on IGF-1 level distribution was the radiation dose to the pituitary gland $(p=0.04)$, while none of the remaining variables showed a significant influence on IGF-1 levels. 
Table 7. GH peaks, number of patients tested, and age and type of GH stimulation tests performed at final height reassessment of GH status

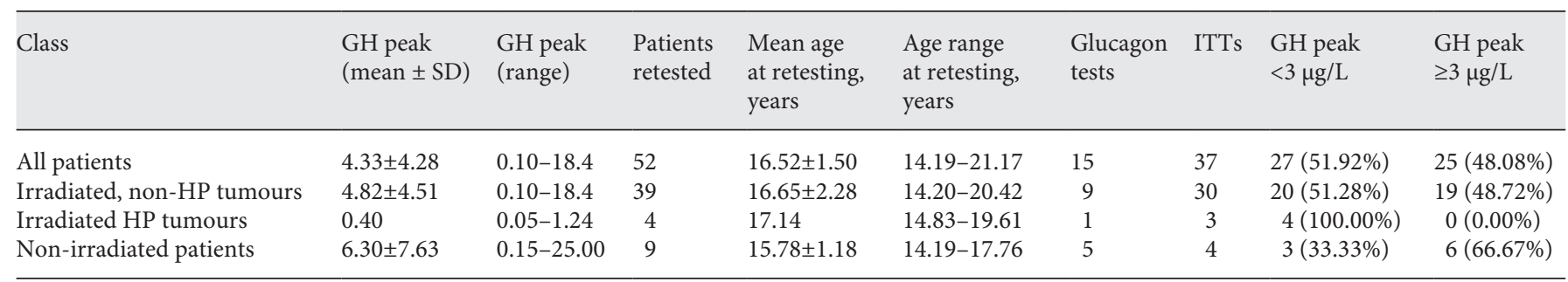

GH, growth hormone; HP, hypothalamic-pituitary; ITTs, insulin tolerance tests; SD, standard deviation.

Fig. 3. Delta height SDS at 1 and 2 years after treatment with $\mathrm{GH}$ and at final height in patients treated with cranial irradiation, craniospinal irradiation, and TBI. GH, growth hormone; SDS, standard deviation score; TBI, total body irradiation.

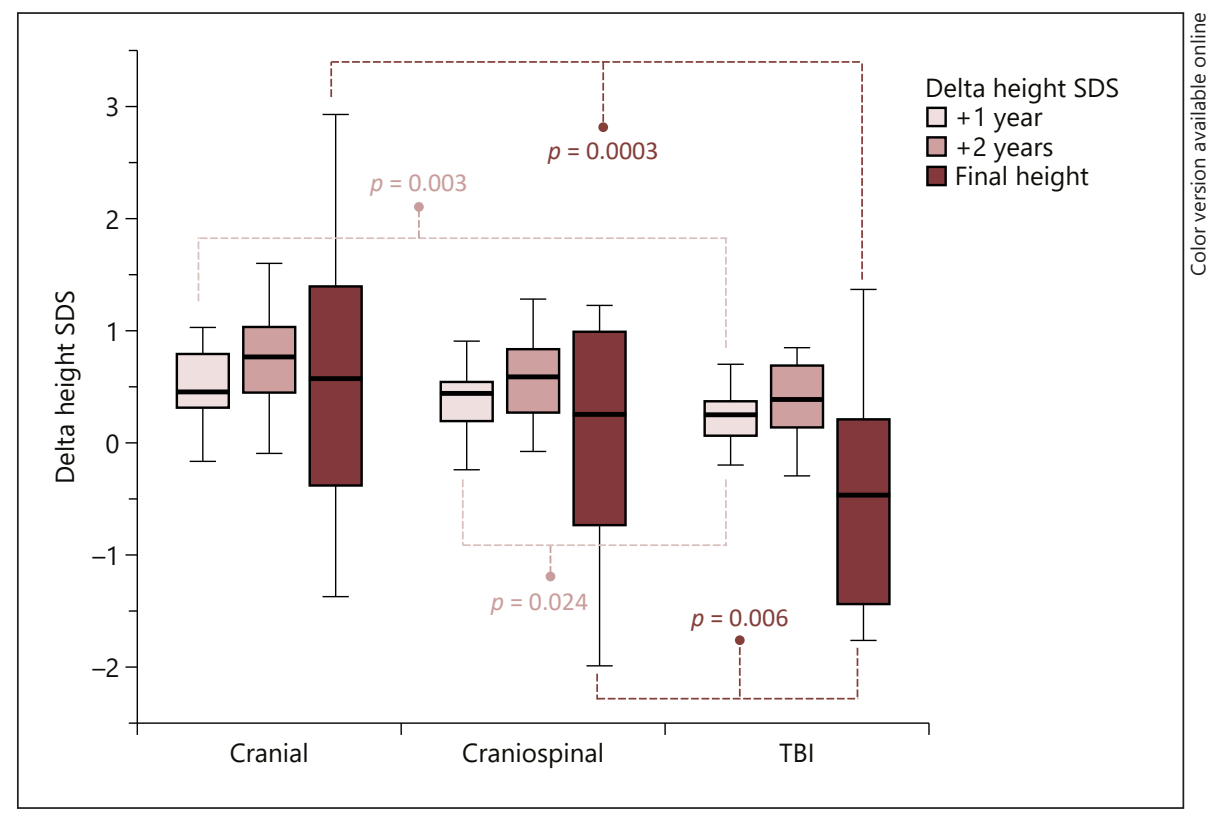

\section{IGF-1 SDS and Delta Height SDS}

We recorded the variations in height SDS as delta height SDS, namely the difference between height SDS at specific time points ( 1 and 2 years after starting rhGH and at final height) and height SDS before starting rhGH treatment.

In our study population, delta height SDS after 1 year of treatment with rhGH was available in 107 patients. Among the 90/158 patients who had already achieved their definitive stature at the moment of data collection, delta height SDS at final height could be estimated in 68 subjects.

Figure 3 shows the values of delta height SDS at 1 and 2 years of treatment and at final height in RT-NHP patients who underwent cranial irradiation, craniospinal irradiation, or TBI.
After 1 year of treatment, the delta height SDS in patients treated with TBI was $0.24 \pm 0.23$, statistically lower than the delta height SDS in patients treated with craniospinal irradiation $(0.38 \pm 0.26, p=0.024)$ and in patients treated with isolated cranial irradiation $(0.51 \pm 0.30, p=$ $0.003)$. In addition, our results showed similar outcomes in patients treated with rhGH at final height reassessment, with delta height SDS after TBI $(-0.48 \pm 0.99)$ being statistically lower than after craniospinal $(0.04 \pm 0.97, p=$ $0.006)$ and cranial $(0.57 \pm 1.03, p=0.0003)$ irradiation.

In the RT-NHP group, baseline IGF-1 SDS levels showed no significant correlation with delta height SDS after 1 year of rhGH (Pearson coefficient 0.03 ) and a very weak negative correlation with delta height SDS at final height (Pearson coefficient $-0.36, p=0.04$ ). 


\section{GH and IGF-1 Status at Final Height}

Ninety of the 158 patients had already achieved final height at the moment of data collection. In 26/90 patients, rhGH was continued in adulthood without reassessing GH status because these subjects showed a severe GHD in childhood and because in consideration of their oncological diagnosis (i.e., tumours with direct pituitary involvement), the probability of achieving a normal peak at final height reassessment was estimated to be very low and retesting was therefore not performed. These patients will undergo retesting of their pituitary function later on in early adulthood under the care of the adults endocrine team. In 52/90 subjects, GH status was reassessed at final height; retesting was performed $6.07 \pm 2.35$ years after starting rhGH treatment (range 2.53-11.28). Finally, the remaining $12 / 90$ patients were not retested for other reasons (3 patients were diagnosed with a relapse of oncological disease, 1 patient deceased before retesting, 4 patients were transferred to other centres before retesting, 2 were not tested due to specific family request, and in 2 patients retesting was scheduled but not performed yet at the moment of data collection).

Fifty of the 52 patients reassessed for GH status at final height had IGF-1 assessment repeated before undergoing GH stimulation test and more than 3 months after rhGH treatment was discontinued; the results are illustrated in Table 7. Eleven out of 11 patients from the whole population and 7 out of the 7 subjects form the RT-NHP group with IGF-1 levels $<-2$ SDS achieved a GH peak $<3 \mu \mathrm{g} / \mathrm{L}$ at final height reassessment. The frequency of IGF-1 levels $<-2$ SDS was statistically higher in patients with a GH peak $<3 \mu \mathrm{g} / \mathrm{L}$ than in those with a GH peak $\geq 3 \mu \mathrm{g} / \mathrm{L}$, both in the whole population (single-tailed Fisher exact test, $p=0.001)$ and in the RT-NHP cohort $(p=0.006)$. None of the subjects who achieved a GH peak $\geq 3 \mu \mathrm{g} / \mathrm{L}$ at final height reassessment presented an IGF-1 SDS $<-2$.

In the RT-NHP cohort, IGF-1 levels $<-2$ SDS had a sensitivity of $35 \%$, a specificity of $100 \%$, a positive predictive value of $100 \%$, and a negative predictive value of $58.1 \%$.

Finally, in the RT-NHP group, we found a statistically significant correlation between pretreatment IGF-1 SDS values determined at diagnosis of GHD in childhood and $\mathrm{GH}$ peaks achieved after dynamic stimulation tests at final height reassessment (Pearson coefficient 0.47, $p=$ 0.0036).

\section{Correlation between IGF-1 SDS and the Number of}

Deficient Pituitary Hormones

In order to identify a possible correlation between lower IGF-1 levels at diagnosis of GHD and an increased risk of developing other pituitary deficiencies, we analysed the correlation between baseline IGF-1 SDS levels and the number of pituitary hormones found to be deficient at the last follow-up in our paediatric endocrine clinic. We only considered patients who had completed their antineoplastic treatment more than 5 years before the moment of data collection, as the onset of several pituitary deficiencies can take many years before becoming overt. In addition, as the diagnosis of luteinizing hormone/follicle-stimulating hormone deficiency (central hypogonadism) is strictly age-dependent, we also excluded patients younger than 15 years at the moment of data collection. Among the 68 patients from the RT-NHP group who fulfilled these criteria, our data did not show a significant statistical correlation between baseline IGF-1 SDS levels and the number of pituitary hormones found to be deficient (Pearson coefficient -0.12). Also, the correlation between the number of pituitary deficiencies and IGF-1 SDS levels at final height reassessment was not significant (Pearson coefficient -0.10).

All the patients who developed deficiency of all anterior and posterior pituitary hormones were diagnosed with a tumour directly involving the HP area (craniopharyngiomas and pituitary germ cell tumours).

\section{Discussion}

Several studies have analysed the predictive accuracy of low IGF-1 levels in mixed cohorts of children diagnosed with GHD due to different aetiologies, and the reported sensitivity of IGF- 1 levels $<-2$ SDS ranged from 32 to $100 \%$ [15-18]. Recently, a wide meta-analysis by Shen et al. [19] found that IGF-1 levels $<-2$ SDS had a pooled sensitivity of $66 \%$ and a pooled specificity of $69 \%$, and the authors concluded that IGF-1 is a useful screening tool in patients at risk of developing GHD. Most studies did not focus on radiation-dependent GHD, and specific figures about the role of low IGF-1 in irradiated CCS were drawn from small samples of patients $[8,10-12]$ and provided conflicting outcomes.

In 1989, Brauner et al. [11] analysed the incidence of GHD in 16 paediatric patients diagnosed with medulloblastoma or ependymoma treated with either cranial or craniospinal radiotherapy. In 13/16 patients the authors found a correlation between IGF-1 values and the response to GH stimulation test. The main limitations of that study are both the small sample size and the lack of age-dependent reference ranges for IGF-1 values. 
Conversely, in two studies performed by Tillmann et al. $[8,9]$ on small cohorts of respectively 12 and 15 irradiated CCS diagnosed with GHD, IGF-1 levels $<-2$ SDS had a sensitivity of 28 and $47 \%$; these data support the contention that IGF-1 is not suitable for predictive screening purposes in irradiated patients. Despite a similar specificity, IGF-1 sensitivity was found to be lower than that reported in other studies where most patients had idiopathic GHD [20, 21].

In another report on 14 irradiated CCS, Achermann et al. [12] found a statistical correlation between IGF-1 levels and $\mathrm{GH}$ peaks recorded during 24-h profiles and suggested that IGF-1 can be considered a good marker of GH dysregulation in irradiated children. Unfortunately, this study neither provided a correlation between GH dynamic tests and IGF-1 levels, nor did it determine its sensibility and specificity in predicting GHD in irradiated children.

We collected data on 158 CCS with GHD and specifically analysed the screening role of low IGF-1 levels in a selected cohort of 117 irradiated subjects with no primary involvement of the HP area (RT-NHP group). To our knowledge, this is the largest available cohort of irradiation-dependent GHD patients on which a specific analysis on IGF-1 sensitivity has been performed to date. Data on these 117 patients allow analysis of the specific impact of radiotherapy on $\mathrm{GH}$ secretion because GHD can be the consequence of either a direct detrimental effect of cancer itself on somatotrophic cells or the final outcome of surgical attempts at removing the tumour when the tumour includes the HP area.

In the RT-NHP group, IGF-1 values $<-2$ SDS had a sensitivity of $31.86 \%$. These data support the hypothesis that IGF-1 is not suitable for screening purposes in irradiated patients and confirm on a wider sample the conclusions drawn by Tillmann et al. $[8,9]$.

Our results showed that the frequency of IGF-1 levels $<-2$ SDS was statistically higher in the paediatric population with severe GHD ( $\mathrm{GH}$ peak $<3 \mu \mathrm{g} / \mathrm{L})$ both in the whole population and in the selected RT-NHP cohort. In the latter, IGF- 1 sensitivity at -2 SDS rose to $43.55 \%$ in patients with severe GHD. A longitudinal study by Nivot et al. [10] about irradiated CCS suggests non-parallel changes in IGF-1 levels and GH peak achieved, with unchanged IGF-1 levels 2 years after irradiation and a progressive decrease in $\mathrm{GH}$ peaks achieved after stimulation tests. Conversely, we found a statistically significant positive correlation between IGF-1 levels and GH peaks in patients with irradiation-dependent GHD. Our results suggest that IGF-1 levels are statistically lower in patients with more severe impairment in GH secretion and that IGF-1 is therefore more accurate as a screening test in patients with more severe irradiation-induced GHD. In summary, despite an overall poor sensitivity in radiationinduced GHD, the diagnostic value of an IGF- $1<-2$ SDS statistically improves in the subcohort of subjects with severe GHD. Given the demonstrated negative correlation between pituitary irradiation and GH peaks achieved after stimulation tests, the use of IGF-1 as a screening tool could be restricted to patients treated with higher cranial radiation doses. These considerations are consistent with the recent recommendations jointly provided by the European Endocrine Society and the Pediatric Endocrine Society concerning the HP disorder experienced by CCS, which state that there is a potential diagnostic reliability of low IGF-1 levels only in patients with severe GHD [7].

In patients diagnosed with GHD due to different aetiologies, the sensitivity of low IGFBP-3 levels has been widely debated: Nunez et al. [22] found a sensitivity of $50 \%$; in the above-mentioned study by Tillmann et al. [8], the sensitivity in non-irradiated GHD subjects was $53 \%$. Conversely, in CCS with irradiation-dependent GHD, the diagnostic accuracy of IGFBP-3 levels $<-2$ SDS was found to be extremely poor $[8,9,12]$, and our analysis supports this outcome (sensitivity in the RT-NHP cohort 7.37\%). We are unable to provide an explanation for the poorer sensitivity of IGFBP-3 in irradiated patients. Some authors suggest that while in rapidly progressing GHD IGFBP-3 levels are markedly reduced due to a premature decrease in GH levels, in patients with slowly progressing GHD (i.e., induced by cranial radiation), compensatory mechanisms take over and maintain adequate IGFBP-3 levels [8].

Our study provided data on the distribution of $\mathrm{GH}$ peaks and IGF-1 values at final height. Despite the low sensitivity of $35 \%$, IGF-1 levels showed a positive predictive value of $100 \%$ at final height reassessment: when a patient diagnosed with childhood GHD underwent a GH dynamic test after achieving final height and stopping $\mathrm{GH}$ replacement therapy, an IGF-1 level <-2 SDS predicted adulthood GHD in $100 \%$ of cases. In a wide study on 90 irradiated CCS by Adan et al. [23], IGF-1 levels <-2 SDS had a sensitivity of $95 \%$ in subjects who achieved a peak $<3 \mu \mathrm{g} / \mathrm{L}$ after a combined arginine and insulin stimulation test at final height reassessment. We are not able to entirely explain this significant discrepancy with the lower sensitivity found in our study, but part of the difference may be due to the fact that the authors used a combined arginine-insulin tolerance test to assess $\mathrm{GH}$ status while we used an ITT $(n=37)$ or a glucagon stimulation test 
$(n=15)$. A cut-off of $3 \mu \mathrm{g} / \mathrm{L}$ following a combined arginine-insulin stimulation test can express a more profound GHD than a similar cut-off with ITT or a glucagon stimulation test. As more severe GHD correlates with lower IGF-1 levels, this may explain the higher sensitivity of low IGF-1 values in this selected cohort of patients. On the other hand, several papers support the hypothesis of a poor sensitivity of IGF-1 in adulthood, in keep with our analysis: in a mixed population of irradiated and non-irradiated patients, Hoffman et al. [24] found a sensitivity of $30 \%$ when the GH peak to diagnose GHD was set at $5 \mu \mathrm{g} / \mathrm{L}$. Some of the discrepancies among the cited studies can be attributed to the timing of the onset of GHD. In a wide study on 74 childhood- and 99 adulthood-onset GHD patients, Attanasio et al. [25] found a remarkably lower diagnostic accuracy of IGF-1 $<-2$ SDS in the latter category and concluded that IGF-1 levels are more frequently reduced in childhood- than in adulthood-onset GHD. As Adan et al. [23] enrolled patients with childhood-onset GHD reassessed at final height while the study by Hoffman et al. [24] mainly dealt with subjects diagnosed in adulthood, this may explain the cited different outcomes in terms of IGF-1 sensitivity.

Our paper analyses the correlation between GH peaks and the estimated pituitary radiation exposure in paediatric patients. Given the anatomical continuity of the hypothalamus with the surrounding cerebral structures, outlining the edges of the hypothalamus may be challenging also for experienced radiologists, and providing an estimation of the hypothalamic mean and maximum radiation dose may produce misleading results. We therefore decided to collect and analyse only the pituitary mean doses, given the small dimensions and well-defined anatomy of this gland. We are aware that the hypothalamus has been widely described as more sensitive to the detrimental effects of cranial irradiation, but our decision was driven by the purpose of providing reproducible and reliable estimations. In our analysis, we confirm the statistically significant negative correlation between the pituitary radiation exposure and GH peaks achieved after dynamic stimulation in childhood. Similar results were drawn by Talvensaari et al. [26] and Schmiegelow et al. [1] on smaller samples of irradiated GHD children and by Adan et al. [23] in 90 young adults at final height retesting. Our analysis extends these results to a wider population of irradiated patients who underwent $\mathrm{GH}$ assessment in childhood, and therefore confirms that this correlation can be already demonstrated $2.06 \pm$ 1.06 years after the end of cranial irradiation.

According to the data collected in the specific RT-NHP cohort, we did not find a statistical correlation between ei-

IGF-1 in Radiation-Induced Growth Hormone Deficiency ther pretreatment or final height IGF-1 level distribution and the number of hormonal deficiencies recorded at last clinical follow-up. In a wide study on 269 adult patients with pituitary disease due to different aetiologies (pituitary tumours, pituitary surgery, congenital HP abnormalities, irradiation, etc.), Tirosh et al. [27] described a good statistical correlation between IGF-1 SDS distribution and the severity of hypopituitarism expressed as the number of altered hormonal axes. We are unable to explain this discrepancy, but it is possible that in this population of adult patients with frequent surgical and/or tumoral involvement of the pituitary, the HP damage was more severe and longer-lasting than in our population of children/young adults with a postradiation impairment of the hypothalamic or pituitary secretion pattern. Our opinion is that low IGF-1 SDS levels may more precisely reflect a severe anatomical HP damage (surgery, tumoral invasion) than a mere functional dysregulation (radiation damage).

Finally, our study allows specific conclusions about the role of IGF-1 in the cohort of leukaemic patients. In irradiated patients diagnosed with acute lymphoblastic leukaemia, IGF-1 levels <-2 SDS had a sensitivity of only $17.86 \%$, and it was even lower (7.14\%) in subjects treated with only $14.4 \mathrm{~Gy}$ as TBI before bone marrow transplantation. Leukaemic patients received lower doses of cranial radiation and therefore developed milder impairment of GH secretion compared to subjects exposed to higher pituitary radiation doses due to brain tumours. As we found a higher sensitivity of low IGF-1 levels in patients with severe GHD, this may explain the poor reliability of IGF-1 as a screening tool in this specific subcohort.

Our study had limitations. Firstly, we had partial data sets due to the retrospective nature of our analysis. Secondly, estimation of the hypothalamic radiation dose may be even more important than pituitary dosimetry in order to predict the detrimental effect of cranial irradiation on GH secretion. Finally, comparison of our cohort with a control group of non-oncological patients diagnosed with GHD due to different aetiologies would have provided more complete information about the specific role of IGF-1 in irradiation-dependent GHD. Future prospective studies could confirm our findings.

\section{Conclusions}

Our analysis demonstrates an overall poor sensitivity of IGF-1 levels $<-2$ SDS in radiation-induced GHD. Sensitivity is higher in patients with severe GHD, while normal plasma IGF-1 concentrations despite a diagnosis of 
GHD are seen most frequently after low radiation doses to the HP area.

In addition, our analysis supports the diagnostic value of IGF-1 at the time of reassessing GH status at final height in patients with established irradiation-induced GHD in childhood: low IGF-1 levels had a positive predictive value of $100 \%$ and - if confirmed by future larger prospective studies - this could allow the possibility of avoiding GH retesting in young adults with low IGF-1 levels after $\mathrm{GH}$ therapy discontinuation.

\section{Statement of Ethics}

The study protocol was conducted ethically in accordance with the World Medical Association Declaration of Helsinki and was approved by the research institute's committee on human research.

\section{Disclosure Statement}

The authors have no conflicts of interest to disclose.

\section{Author Contributions}

A. Cattoni: collection, interpretation, and analysis of data, writing of the paper, and approval of the final version. E. Clarke: dosimetric analysis of radiotherapy exposure for enrolled patients and approval of the final version. A. Albanese: conception and design of the study, supervision of data collection and analysis, and approval of the final version.

\section{References}

1 Schmiegelow M, Lassen S, Weber L, Poulsen HS, Hertz H, Müller J. Dosimetry and growth hormone deficiency following cranial irradiation of childhood brain tumors. Med Pediatr Oncol. 1999 Dec;33(6):564-71.

2 Chieng PU, Huang TS, Chang CC, Chong $\mathrm{PN}$, Tien RD, Su CT. Reduced hypothalamic blood flow after radiation treatment of nasopharyngeal cancer: SPECT studies in $34 \mathrm{pa}-$ tients. AJNR Am J Neuroradiol. 1991 JulAug;12(4):661-5.

3 Ryalls M, Spoudeas HA, Hindmarsh PC, Matthews DR, Tait DM, Meller ST, et al. Shortterm endocrine consequences of total body irradiation and bone marrow transplantation in children treated for leukemia. J Endocrinol. 1993 Feb;136(2):331-8.

4 Chrousos GP, Poplack D, Brown T, O'Neill D, Schwade J, Bercu BB. Effects of cranial radiation on hypothalamic-adenohypophyseal function: abnormal growth hormone secretory dynamics. J Clin Endocrinol Metab. 1982 Jun;54(6):1135-9.

5 Darzy KH, Pezzoli SS, Thorner MO, Shalet $\mathrm{SM}$. The dynamics of growth hormone (GH) secretion in adult cancer survivors with severe GH deficiency acquired after brain irradiation in childhood for nonpituitary brain tumors: evidence for preserved pulsatility and diurnal variation with increased secretory disorderliness. J Clin Endocrinol Metab. 2005 May;90(5):2794-803.

6 Children's Oncology Group. Long-Term Follow-Up Guidelines for Survivors of Childhood, Adolescent, and Young Adult Cancers. www.survivorshipguidelines.org.
7 Sklar CA, Antal Z, Chemaitilly W, Cohen LE, Follin C, Meacham LR, et al. HypothalamicPituitary and Growth Disorders in Survivors of Childhood Cancer: An Endocrine Society Clinical Practice Guideline. J Clin Endocrinol Metab. 2018 Aug;103(8):2761-84.

8 Tillmann V, Buckler JM, Kibirige MS, Price DA, Shalet SM, Wales JK, et al. Biochemical tests in the diagnosis of childhood growth hormone deficiency. J Clin Endocrinol Metab. 1997 Feb;82(2):531-5.

9 Tillmann V, Shalet SM, Price DA, Wales JK, Pennells L, Soden J, et al. Serum insulin-like growth factor-I, IGF binding protein-3 and IGFBP-3 protease activity after cranial irradiation. Horm Res. 1998;50(2):71-7.

10 Nivot S, Benelli C, Clot JP, Saucet C, Adan L, Souberbielle JC, et al. Nonparallel changes of growth hormone (GH) and insulin-like growth factor-I, insulin-like growth factor binding protein-3, and GH-binding protein, after craniospinal irradiation and chemotherapy. J Clin Endocrinol Metab. 1994 Mar; 78(3):597-601.

11 Brauner R, Rappaport R, Prevot C, Czernichow P, Zucker JM, Bataini P, et al. A prospective study of the development of growth hormone deficiency in children given cranial irradiation, and its relation to statural growth. J Clin Endocrinol Metab. 1989 Feb;68(2): 346-51.

12 Achermann JC, Hindmarsh PC, Brook CG. The relationship between the growth hormone and insulin-like growth factor axis in long-term survivors of childhood brain tumours. Clin Endocrinol (Oxf). 1998 Nov; 49(5):639-45.
13 Growth Hormone Research Society. Consensus guidelines for the diagnosis and treatment of growth hormone (GH) deficiency in childhood and adolescence: summary statement of the GH Research Society. GH Research Society. J Clin Endocrinol Metab. 2000 Nov; 85(11):3990-3.

14 National Institute for Health and Clinical Excellence. Human growth hormone (somatropin) for the treatment of growth failure in children. technology appraisal guidance [TA188]. https://www.nice.org.uk/guidance/ ta188.

15 Audí L, Antonia Llopis M, Luisa Granada M, Hermoso F, del Valle J, Dolores RodríguezArnao M, et al.; Grupo Español de Estudio de la Talla Baja. [Low sensitivity of IGF-I, IGFBP-3 and urinary GH in the diagnosis of growth hormone insufficiency in slowlygrowing short-statured boys. Grupo Español de Estudio de la Talla Baja]. Med Clin (Barc). 2001 Jan;116(1):6-11.

16 Boquete HR, Sobrado PG, Fideleff HL, Sequera AM, Giaccio AV, Suárez MG, et al. Evaluation of diagnostic accuracy of insulinlike growth factor (IGF)-I and IGF-binding protein-3 in growth hormone-deficient children and adults using ROC plot analysis. J Clin Endocrinol Metab. 2003 Oct;88(10): 4702-8.

17 Cianfarani S, Tondinelli T, Spadoni GL, Scirè G, Boemi S, Boscherini B. Height velocity and IGF-I assessment in the diagnosis of childhood onset GH insufficiency: do we still need a second GH stimulation test? Clin Endocrinol (Oxf). 2002 Aug;57(2):161-7. 
18 Jaruratanasirikul S, Leethanaporn K, Sriplung $\mathrm{H}$. The usefulness of serum insulin-like growth factor-1 (IGF-1) and insulin-like growth factor binding protein-3 (IGFBP-3) for evaluation of children with short stature. J Med Assoc Thai. 2000 Jun;83(6):619-26.

19 Shen Y, Zhang J, Zhao Y, Yan Y, Liu Y, Cai J. Diagnostic value of serum IGF-1 and IGFBP-3 in growth hormone deficiency: a systematic review with meta-analysis. Eur J Pediatr. 2015 Apr;174(4):419-27.

20 Blum WF, Ranke MB, Kietzmann K, Gauggel E, Zeisel HJ, Bierich JR. A specific radioimmunoassay for the growth hormone $(\mathrm{GH})$ dependent somatomedin-binding protein: its use for diagnosis of GH deficiency. J Clin Endocrinol Metab. 1990 May;70(5):1292-8.

21 Rosenfeld RG, Wilson DM, Lee PD, Hintz RL. Insulin-like growth factors I and II in evaluation of growth retardation. J Pediatr. 1986 Sep;109(3):428-33.
22 Nunez SB, Municchi G, Barnes KM, Rose SR. Insulin-like growth factor I (IGF-I) and IGFbinding protein-3 concentrations compared to stimulated and night growth hormone in the evaluation of short children - a clinical research center study. J Clin Endocrinol Metab. 1996 May;81(5):1927-32.

23 Adan L, Trivin C, Sainte-Rose C, Zucker JM, Hartmann O, Brauner R. GH deficiency caused by cranial irradiation during childhood: factors and markers in young adults. J Clin Endocrinol Metab. 2001 Nov;86(11): 5245-51.

24 Hoffman DM, O'Sullivan AJ, Baxter RC, Ho KK. Diagnosis of growth-hormone deficiency in adults. Lancet. 1994 Apr;343(8905):1064Birkett MA, Bates PC, Valk NK, et al.; Adult Growth Hormone Deficiency Study Group. Adult growth hormone (GH)-deficient patients demonstrate heterogeneity between childhood onset and adult onset before and during human GH treatment. J Clin Endocrinol Metab. 1997 Jan;82(1):82-8.

26 Talvensaari KK, Lanning M, Pääkkö E, Tapanainen P, Knip M. Pituitary size assessed with magnetic resonance imaging as a measure of growth hormone secretion in long term survivors of childhood cancer. J Clin Endocrinol Metab. 1994 Oct;79(4):1122-7.

27 Tirosh A, Toledano Y, Masri-Iraqi H, Eizenberg Y, Tzvetov G, Hirsch D, et al. IGF-I levels reflect hypopituitarism severity in adults with pituitary dysfunction. Pituitary. 2016 Aug; 19(4):399-406 